\title{
On the packing chromatic number of square and hexagonal lattice*
}

\author{
Danilo Korže \\ FERI, University of Maribor, Smetanova 17, SI-2000 Maribor, Slovenia \\ Aleksander Vesel ${ }^{\dagger}$ \\ Faculty of Natural Sciences and Mathematics, University of Maribor \\ Koroška cesta 160, SI-2000 Maribor, Slovenia
}

Received 20 October 2011, accepted 31 October 2012, published online 7 January 2013

\begin{abstract}
The packing chromatic number $\chi_{\rho}(G)$ of a graph $G$ is the smallest integer $k$ such that the vertex set $V(G)$ can be partitioned into disjoint classes $X_{1}, \ldots, X_{k}$, with the condition that vertices in $X_{i}$ have pairwise distance greater than $i$. We show that the packing chromatic number for the hexagonal lattice $\mathcal{H}$ is 7 . We also investigate the packing chromatic number for infinite subgraphs of the square lattice $\mathbb{Z}^{2}$ with up to 13 rows. In particular, we establish the packing chromatic number for $P_{6} \square \mathbb{Z}$ and provide new upper bounds on these numbers for the other subgraphs of interest. Finally, we explore the packing chromatic number for some infinite subgraphs of $\mathbb{Z}^{2} \square P_{2}$. The results are partially obtained by a computer search.
\end{abstract}

Keywords: Packing chromatic number, hexagonal lattice, square lattice, computer search.

Math. Subj. Class.: 05C70, 05C85

\section{Introduction and preliminaries}

The packing coloring was introduced by Goddard et al. in [6] under the name broadcast coloring. The concept comes from the regulations concerning the assignment of broadcast frequencies to radio stations. In particular, two radio stations which are assigned the same frequency must be placed sufficiently far apart so that neither broadcast interferes with the reception of the other. Moreover, the geographical distance between two radio stations

\footnotetext{
* Supported by the Ministry of Science of Slovenia under the grant 0101-P-297.

${ }^{\dagger}$ Corresponding author.

E-mail addresses: korze@uni-mb.si (Danilo Korže), vesel@uni-mb.si (Aleksander Vesel)
} 
which are assigned the same frequency is directly related to the power of their broadcast signals. These frequency restrictions have inspired the graphical coloring problem defined below.

A $k$-coloring of a graph $G$ is a function $f$ from $V(G)$ onto a set $C=\{1,2, \ldots, k\}$ (with no additional constraints). The elements of $C$ are called colors. Let $X_{i}$ denote the set of vertices with the image (color) $i$. Note that $X_{1}, \ldots, X_{k}$ is partition of the vertex set of $G$ into disjoint (color) classes.

Let $X_{1}, \ldots, X_{k}$ be a partition of the vertex set of $G$ with respect to the following constraints: each color class $X_{i}$ is a set of vertices with the property that any distinct pair $u, v \in X_{i}$ satisfies $d_{G}(u, v)>i$. Here $d_{G}(u, v)$ denotes the usual shortest path distance between $u$ and $v$. Then $X_{i}$ is said to be an $i$-packing, while such a partition is called a packing $k$-coloring. The smallest integer $k$ for which there exists a packing $k$-coloring of $G$ is called the packing chromatic number of $G$ and it is denoted by $\chi_{\rho}(G)$.

Let $G=(V, E)$ be a graph. A walk is a sequence of vertices $v_{1}, v_{2}, \ldots, v_{k}$ and edges $v_{i} v_{i+1}, 1 \leq i \leq k-1$. A path on $n$ vertices is a walk on $n$ distinct vertices and denoted $P_{n}$. A walk is closed if $v_{1}=v_{n}$. A closed walk in which all vertices (except the first and the last) are different, is a cycle. The cycle on $n$ vertices is denoted $C_{n}$. For $u, v \in V(G)$, $d_{G}(u, v)$ or $d(u, v)$ denotes the length of the shortest walk (i.e., the number of edges on the shortest walk) in $G$ from $u$ to $v$. These definitions extend naturally to directed graphs.

A set $S \subseteq V(G)$ is independent if $x y \notin E(G)$ for any pair of vertices $x, y \in S$. Cardinality of a largest independent set $S$ of $G$ is the independence number $\alpha(G)$ of $G$.

This paper studies the packing chromatic number of hexagonal lattice and of some infinite subgraphs of square lattice. Section 2 contains the search for the lower bound on the packing chromatic number in hexagonal lattice. The bound is obtained by a computer program using the dynamic approach for computing graph invariants, as described in the first part of the section. Section 3 discusses the packing chromatic number for some infinite subgraphs of the square lattice. We establish the packing chromatic number for $P_{6} \square \mathbb{Z}$ and provide upper bounds on these numbers for $P_{n} \square \mathbb{Z}$, where $7 \leq n \leq 13$. We conclude the paper with the packing chromatic number for $C_{4} \square \mathbb{Z}$ as well as with some partial results on upper bounds for some infinite subgraphs of $\mathbb{Z}^{2} \square P_{2}$ provided in Section 4 .

The results in our paper were partially obtained by computers, mainly in Windows environment, but some also using Linux Ubuntu operating system. The machines used for computations were also diverse: Intel i7 930 based personal computer, Intel Q9400 based machine and a computer cluster (with up to 24 processor cores). All computations were carried out during six months, starting in the middle of 2010. The development environment and class libraries Lazarus (version of Pascal language) were used to write all necessary programs.

\section{Hexagonal lattice}

The hexagonal lattice $\mathcal{H}$ plays a crucial role in many network applications, particularly in frequency assignments, e.g. see [5]. It was proved by Brešar et al. [1] that the packing chromatic number of the infinite hexagonal lattice lies between 6 and 8 . The result was improved by Fiala et al. [3], where the packing 7-coloring of the hexagonal lattice is presented.

We show in this section that actual lower bound on the packing chromatic number of the infinite hexagonal lattice is 7 and therefore $\chi_{\rho}(\mathcal{H})=7$. 
We now present the algorithms, that have been used to provide the main result. We first describe the concept needed to describe our computer checking. The idea is introduced in [9] in a more general framework, but for our purposes the following description will be sufficient.

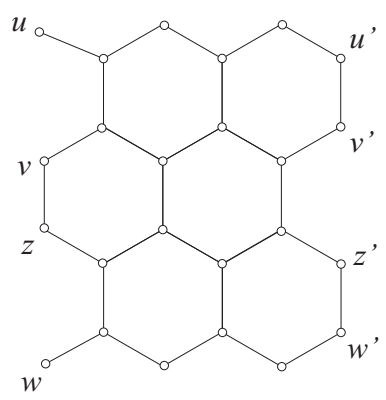

Figure 1: Graph $H_{1}$.

Observe first the graph $H_{1}$ depicted in Fig. 1. We construct $H_{i}$ for $i>1$ as follows. Take the graph which is composed of an isomorphic copy of $H_{i-1}$ and of an isomorphic copy of $H_{1}$. Then add additional four edges that connect vertices $u^{\prime}, v^{\prime}, w^{\prime}$, and $z^{\prime}$ of the last added copy of $H_{1}$ in $H_{i-1}$ with the vertices $u, v, w$, and $z$ of the new copy of $H_{1}$. As an example see Fig. 2 where $H_{2}$ is depicted.

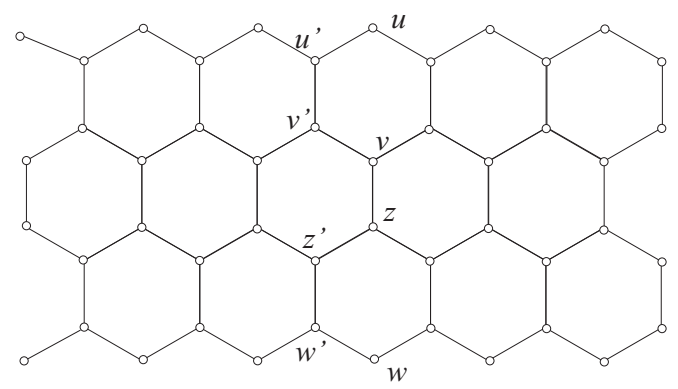

Figure 2: Graph $\mathrm{H}_{2}$.

Obviously, $H_{i}$ is a subgraph of $\mathcal{H}$ for $i \geq 1$.

We next define a directed graph $D_{k}$ as follows.

The vertices of $D_{k}$ are all packing $k$-colorings of $H_{1}$. Let $u$ and $v$ be two distinct vertices of $D_{k}$. Then $\widetilde{u v}$ denotes a $k$-coloring of $H_{2}$ such that $u$ and $v$ induce the respective packing $k$-coloring of the first and the second copy of $H_{1}$. Note that $\widetilde{u v}$ need not to be a packing $k$-coloring of $H_{2} . u v$ is an arc in $D_{k}$ if and only if $\widetilde{u v}$ is a packing $k$-coloring of $\mathrm{H}_{2}$.

Lemma 2.1. Let $k \leq 6$. Then $H_{i}$ admits a packing $k$-coloring if and only if $D_{k}$ possesses a walk $P=v_{1}, v_{2}, \ldots, v_{i}$ with $v_{j}$ corresponding to the $j$-th copy of $H_{1}$. 
Proof. Suppose first that $D_{k}$ possesses a walk $P=v_{1}, v_{2}, \ldots, v_{i}$. If $i=2$, then $P$ is an arc from $v_{1}$ to $v_{2}$ in $D_{k}$ and the claim is obvious. Let then $i>2$. Suppose the claim holds for $P^{\prime}=v_{1}, v_{2}, \ldots, v_{i-1}$, i.e. $H_{i-1}$ admits a packing $k$-coloring. Since $P$ has an arc from $v_{i-1}$ to $v_{i}$, the corresponding colorings induce a packing $k$-coloring in a copy of $H_{2}$ that corresponds to $v_{i-1}$ and $v_{i}$. In order to see that the assertion holds, note that the distance between a vertex of a copy of $H_{1}$ that corresponds to $v_{i}$ and a vertex of a copy of $H_{1}$ that corresponds to $v_{i-2}$ is at least 7 .

Suppose now that $H_{i}$ admits a packing $k$-coloring. If $i=2$, then by definition of $D_{k}$ a packing $k$-coloring of $H_{2}$ induce an arc in $D_{k}$. Let then $i>2$. Note that $H_{i}$ is composed of an isomorphic copy of $H_{i-1}$, say $X$, and of an isomorphic copy of $H_{1}$, say $Y$. $Y$ is connected in $H_{i}$ to an isomorphic copy of $H_{1}$, say $Z$. Suppose the claim holds for $H_{i-1}$ and let $P^{\prime}=v_{1}, v_{2}, \ldots, v_{i-1}$ denote a walk in $D_{k}$ that corresponds to $X$. Since $H_{i}$ admits a packing $k$-coloring, $Y$ induces a packing $k$-coloring of $H_{1}$, say $v_{i}$. $Y$ and $Z$ together induce a packing $k$-coloring of $H_{2}$ and therefore $v_{i-1} v_{i}$ forms an arc in $D_{k}$. Then $P=v_{1}, v_{2}, \ldots, v_{i-1}, v_{i}$ is a walk in $D_{k}$ and the proof is complete.

Lemma 2.2. Let $k \leq 6$. Then $\mathcal{H}$ admits a packing $k$-coloring only if $D_{k}$ contains a closed directed walk.

Proof. Let $\mathcal{H}$ for a given $k$ admit a packing $k$-coloring denoted $f$. Suppose that $D_{k}$ is acyclic. Since $H_{1}$ is finite, there is obviously only a finite number of vertices (packing $k$-colorings of $H_{1}$ ) in $D_{k}$, say $n_{k}$. Let then $d<n_{k}$ denotes the length of a longest directed path in $D_{k}$. Take now a subgraph of $\mathcal{H}$ isomorphic to $H_{d+2}$. A restriction of $f$ to $H_{d+2}$ is obviously a packing $k$-coloring of $H_{d+2}$. From Lemma 2.1 it follows that $H_{d+2}$ admits a packing $k$-coloring if and only if $D_{k}$ possesses a walk $P=v_{1}, v_{2}, \ldots, v_{d+2}$ with $v_{j}$ corresponding to a packing $k$-coloring of the $j$-th copy of $H_{1}$. But since $D_{k}$ is acyclic, the length of the longest walk in $D_{k}$ is at most $d$ and we obtain a contradiction.

Theorem 2.3. $\chi_{\rho}(\mathcal{H})=7$.

Proof. Since it is proved in [1] that the packing chromatic number of the infinite hexagonal lattice is at least 6 and since in [3] a coloring of the hexagonal lattice using 7 colors is presented, we have to show that $\mathcal{H}$ does not admit a packing 6 -coloring.

We first constructed the graph $D_{6}$ by using a computer program. The graph consists of 26660 vertices with a maximum output degree of 37 (see also the concluding remark). By the depth first search algorithm we next established that $D_{6}$ is an acyclic graph. From Lemma 2.2 then it follows that the hexagonal lattice cannot admit a packing 6 -coloring. This assertion completes the proof.

An alternative approach to prove Theorem 2.3 is to use a naive brute force search for a large enough subgraph of $\mathcal{H}$. The approach used in the proof Theorem 2.3 , however, is potentially much more interesting and utile in order to search for the packing chromatic number in other families of graphs since it uses the packing $k$-colorings of a relatively small graph.

\section{Square lattice}

Cartesian product of graphs provide a setting which has been widely used in designing large scale computer systems and interconnection networks. The Cartesian product of graphs $G$ 
and $H$ is the graph $G \square H$ with vertex set $V(G) \times V(H)$ and $\left(x_{1}, x_{2}\right)\left(y_{1}, y_{2}\right) \in E(G \square H)$ whenever $x_{1} y_{1} \in E(G)$ and $x_{2}=y_{2}$, or $x_{2} y_{2} \in E(H)$ and $x_{1}=y_{1}$. The Cartesian product is commutative and associative, having the trivial graph as a unit, cf. [8]. The subgraph of $G \square H$ induced by $u \times V(H)$ is isomorphic to $H$ and it is called an $H$-fiber.

It will be convenient to view the square lattice as the Cartesian product of two infinite paths, i.e $\mathbb{Z} \square \mathbb{Z}$.

Goddard et al. [6] determined the packing chromatic number for infinite subgraphs of the square lattice $\mathbb{Z}^{2}$ with up to 5 rows. In the same paper the question of determining the packing chromatic number of the infinite square lattice was posed. The best upper bound 17 was given by Holub and Soukal [7], while the best lower bound 12 was determined by Ekstein et al. [2].

We have considered infinite subgraphs of $\mathbb{Z} \square \mathbb{Z}$ with up to 13 rows. The main results are summarized in the following proposition.

\section{Proposition 3.1.}

(i) $\chi_{\rho}\left(P_{6} \square \mathbb{Z}\right)=10$,

(ii) $\chi_{\rho}\left(P_{7} \square \mathbb{Z}\right) \leq 11$,

(iii) $\chi_{\rho}\left(P_{8} \square \mathbb{Z}\right) \leq 12$,

(iv) $\chi_{\rho}\left(P_{9} \square \mathbb{Z}\right) \leq 13$,

(v) $\chi_{\rho}\left(P_{10} \square \mathbb{Z}\right) \leq 14$,

(vi) $\chi_{\rho}\left(P_{11} \square \mathbb{Z}\right) \leq 14$,

(vii) $\chi_{\rho}\left(P_{12} \square \mathbb{Z}\right) \leq 15$.

(viii) $\chi_{\rho}\left(P_{13} \square \mathbb{Z}\right) \leq 15$.

Proof. Note first that if $f$ is a packing $k$-coloring of $P_{n} \square C_{\ell}, k<\ell$, then we can construct from $f$ a packing $k$-coloring of $P_{n} \square P_{m}$ for every $m$. One can use $f$ to color every $P_{n}$-fibre $\left(u_{j} \times P_{n}\right)$ of $P_{n} \square P_{m}$ in the same way as the $P_{n}$-fibre $\left(v_{j} \bmod \ell \times P_{n}\right)$ of $P_{n} \square C_{\ell}$

$\begin{array}{rrrrrrrrrrrrrr}1 & 2 & 1 & 3 & 1 & 2 & 1 & 3 & 1 & 2 & 1 & 3 & 1 & 10 \\ 3 & 1 & 4 & 1 & 8 & 1 & 5 & 1 & 9 & 1 & 4 & 1 & 2 & 1 \\ 1 & 6 & 1 & 2 & 1 & 3 & 1 & 2 & 1 & 3 & 1 & 7 & 1 & 5 \\ 2 & 1 & 3 & 1 & 7 & 1 & 4 & 1 & 6 & 1 & 2 & 1 & 3 & 1 \\ 1 & 9 & 1 & 5 & 1 & 2 & 1 & 3 & 1 & 5 & 1 & 8 & 1 & 4 \\ 3 & 1 & 2 & 1 & 3 & 1 & 10 & 1 & 2 & 1 & 3 & 1 & 2 & 1\end{array}$

Figure 3: A packing 10-coloring of $P_{6} \square C_{14}$

$\begin{array}{rrrrrrrrrrrrrrrr}1 & 2 & 1 & 3 & 1 & 2 & 1 & 3 & 1 & 2 & 1 & 3 & 1 & 4 & 1 & 5 \\ 3 & 1 & 6 & 1 & 4 & 1 & 7 & 1 & 5 & 1 & 6 & 1 & 2 & 1 & 7 & 1 \\ 1 & 8 & 1 & 2 & 1 & 3 & 1 & 2 & 1 & 3 & 1 & 9 & 1 & 3 & 1 & 2 \\ 4 & 1 & 3 & 1 & 5 & 1 & 10 & 1 & 4 & 1 & 2 & 1 & 5 & 1 & 11 & 1 \\ 1 & 2 & 1 & 9 & 1 & 2 & 1 & 3 & 1 & 8 & 1 & 3 & 1 & 2 & 1 & 3 \\ 5 & 1 & 7 & 1 & 3 & 1 & 6 & 1 & 2 & 1 & 7 & 1 & 4 & 1 & 6 & 1 \\ 1 & 3 & 1 & 2 & 1 & 4 & 1 & 5 & 1 & 3 & 1 & 2 & 1 & 3 & 1 & 2\end{array}$

Figure 4: A packing 11-coloring of $P_{7} \square C_{16}$ 


$\begin{array}{rrrrrrrrrrrrrr}1 & 2 & 1 & 3 & 1 & 2 & 1 & 3 & 1 & 2 & 1 & 3 & 1 & 4 \\ 3 & 1 & 5 & 1 & 4 & 1 & 10 & 1 & 11 & 1 & 5 & 1 & 2 & 1 \\ 1 & 8 & 1 & 2 & 1 & 3 & 1 & 2 & 1 & 3 & 1 & 6 & 1 & 9 \\ 2 & 1 & 3 & 1 & 6 & 1 & 5 & 1 & 4 & 1 & 7 & 1 & 3 & 1 \\ 1 & 4 & 1 & 7 & 1 & 2 & 1 & 3 & 1 & 2 & 1 & 12 & 1 & 5 \\ 3 & 1 & 2 & 1 & 3 & 1 & 9 & 1 & 8 & 1 & 3 & 1 & 2 & 1 \\ 1 & 11 & 1 & 5 & 1 & 4 & 1 & 2 & 1 & 5 & 1 & 4 & 1 & 10 \\ 2 & 1 & 3 & 1 & 2 & 1 & 3 & 1 & 6 & 1 & 2 & 1 & 3 & 1\end{array}$

Figure 5: A packing 12-coloring of $P_{8} \square C_{14}$

$\begin{array}{rrrrrrrrrrrrrrrrrrrr}1 & 2 & 1 & 3 & 1 & 2 & 1 & 3 & 1 & 2 & 1 & 3 & 1 & 2 & 1 & 3 & 1 & 2 & 1 & 3 \\ 4 & 1 & 5 & 1 & 6 & 1 & 4 & 1 & 5 & 1 & 7 & 1 & 4 & 1 & 5 & 1 & 6 & 1 & 7 & 1 \\ 1 & 3 & 1 & 2 & 1 & 3 & 1 & 2 & 1 & 3 & 1 & 2 & 1 & 3 & 1 & 2 & 1 & 3 & 1 & 2 \\ 12 & 1 & 8 & 1 & 7 & 1 & 10 & 1 & 11 & 1 & 6 & 1 & 9 & 1 & 13 & 1 & 4 & 1 & 5 & 1 \\ 1 & 2 & 1 & 3 & 1 & 2 & 1 & 3 & 1 & 2 & 1 & 3 & 1 & 2 & 1 & 3 & 1 & 2 & 1 & 3 \\ 6 & 1 & 4 & 1 & 9 & 1 & 5 & 1 & 4 & 1 & 8 & 1 & 5 & 1 & 7 & 1 & 10 & 1 & 11 & 1 \\ 1 & 3 & 1 & 2 & 1 & 3 & 1 & 2 & 1 & 3 & 1 & 2 & 1 & 3 & 1 & 2 & 1 & 3 & 1 & 2 \\ 7 & 1 & 5 & 1 & 13 & 1 & 6 & 1 & 7 & 1 & 12 & 1 & 4 & 1 & 6 & 1 & 5 & 1 & 4 & 1 \\ 1 & 2 & 1 & 3 & 1 & 2 & 1 & 3 & 1 & 2 & 1 & 3 & 1 & 2 & 1 & 3 & 1 & 2 & 1 & 3\end{array}$

Figure 6: A packing 13-coloring of $P_{9} \square C_{20}$

In order to obtained the upper bounds, we therefore first tried to find a packing $k$ coloring of $P_{n} \square C_{m}$ for every $n$ of the interest with $k$ (and $m$ ) being as small as possible.

The obtained colorings for $n \in\{6,7,8,9,11,13\}$ are depicted in Figs. 3 - 8, while a packing 14-coloring of $P_{10} \square C_{16}$ and a packing 15-coloring of $P_{12} \square C_{16}$ can be obtained from the first 10 rows of the packing 14-coloring of $P_{11} \square C_{16}$ depicted in Fig. 7 and the first 12 rows of the packing 15-coloring of $P_{13} \square C_{16}$ depicted in Fig. 8, respectively.

In order to provide the lower bound for $\chi_{\rho}\left(P_{6} \square \mathbb{Z}\right)$ we applied the backtracking search, e.g. see [10], adapted to packing colorings. Since the procedure did not find a packing 9-coloring in $\chi_{\rho}\left(P_{6} \square P_{12}\right)$, the assertion follows.

Results in Proposition 3.1 provide general upper bounds for infinite families of Cartesian products of two paths. For some graphs of these families however, better bounds or even the exact numbers can be computed. The results are depicted on the web page presented in the concluding remark. We again applied the backtracking search, which it is guaranteed to find a solution, if one exits, but it is relatively time consuming and therefore not usable for larger graphs.

The colorings depicted in Figs. 3 - 8 have something in common: every second vertex in a row (column) is colored by the color 1 . We therefore conjecture, that if a packing $k$ coloring of $P_{n} \square P_{m}$ exists, one can always find a packing $k$-coloring such that the class $X_{1}$ is distributed as described above. This conjecture is formally stated below.

Conjecture 3.2. Let $n \geq 4$ and let $\chi_{\rho}\left(P_{m} \square P_{n}\right)=k$. Then exists a packing $k$-coloring of $P_{m} \square P_{n}$ with $\left|X_{1}\right|=\alpha\left(P_{m} \square P_{n}\right)=\left\lceil\frac{n m}{2}\right\rceil$. 


$\begin{array}{rrrrrrrrrrrrrrrr}1 & 2 & 1 & 3 & 1 & 2 & 1 & 3 & 1 & 2 & 1 & 3 & 1 & 2 & 1 & 3 \\ 4 & 1 & 5 & 1 & 8 & 1 & 4 & 1 & 6 & 1 & 7 & 1 & 5 & 1 & 12 & 1 \\ 1 & 3 & 1 & 2 & 1 & 3 & 1 & 2 & 1 & 3 & 1 & 2 & 1 & 3 & 1 & 2 \\ 7 & 1 & 9 & 1 & 13 & 1 & 5 & 1 & 10 & 1 & 11 & 1 & 4 & 1 & 6 & 1 \\ 1 & 2 & 1 & 3 & 1 & 2 & 1 & 3 & 1 & 2 & 1 & 3 & 1 & 2 & 1 & 3 \\ 5 & 1 & 4 & 1 & 6 & 1 & 7 & 1 & 4 & 1 & 5 & 1 & 8 & 1 & 14 & 1 \\ 1 & 3 & 1 & 2 & 1 & 3 & 1 & 2 & 1 & 3 & 1 & 2 & 1 & 3 & 1 & 2 \\ 10 & 1 & 11 & 1 & 5 & 1 & 12 & 1 & 9 & 1 & 6 & 1 & 7 & 1 & 4 & 1 \\ 1 & 2 & 1 & 3 & 1 & 2 & 1 & 3 & 1 & 2 & 1 & 3 & 1 & 2 & 1 & 3 \\ 6 & 1 & 7 & 1 & 4 & 1 & 8 & 1 & 5 & 1 & 4 & 1 & 13 & 1 & 5 & 1 \\ 1 & 3 & 1 & 2 & 1 & 3 & 1 & 2 & 1 & 3 & 1 & 2 & 1 & 3 & 1 & 2\end{array}$

Figure 7: A packing 14-coloring of $P_{11} \square C_{16}$

$\begin{array}{rrrrrrrrrrrrrrrr}1 & 2 & 1 & 3 & 1 & 2 & 1 & 3 & 1 & 2 & 1 & 3 & 1 & 2 & 1 & 3 \\ 4 & 1 & 5 & 1 & 8 & 1 & 14 & 1 & 9 & 1 & 6 & 1 & 7 & 1 & 12 & 1 \\ 1 & 3 & 1 & 2 & 1 & 3 & 1 & 2 & 1 & 3 & 1 & 2 & 1 & 3 & 1 & 2 \\ 6 & 1 & 7 & 1 & 4 & 1 & 15 & 1 & 5 & 1 & 4 & 1 & 10 & 1 & 5 & 1 \\ 1 & 2 & 1 & 3 & 1 & 2 & 1 & 3 & 1 & 2 & 1 & 3 & 1 & 2 & 1 & 3 \\ 11 & 1 & 9 & 1 & 5 & 1 & 6 & 1 & 7 & 1 & 8 & 1 & 13 & 1 & 4 & 1 \\ 1 & 3 & 1 & 2 & 1 & 3 & 1 & 2 & 1 & 3 & 1 & 2 & 1 & 3 & 1 & 2 \\ 5 & 1 & 4 & 1 & 10 & 1 & 12 & 1 & 4 & 1 & 5 & 1 & 6 & 1 & 7 & 1 \\ 1 & 2 & 1 & 3 & 1 & 2 & 1 & 3 & 1 & 2 & 1 & 3 & 1 & 2 & 1 & 3 \\ 8 & 1 & 6 & 1 & 7 & 1 & 5 & 1 & 11 & 1 & 9 & 1 & 4 & 1 & 14 & 1 \\ 1 & 3 & 1 & 2 & 1 & 3 & 1 & 2 & 1 & 3 & 1 & 2 & 1 & 3 & 1 & 2 \\ 4 & 1 & 5 & 1 & 13 & 1 & 4 & 1 & 6 & 1 & 7 & 1 & 5 & 1 & 15 & 1 \\ 1 & 2 & 1 & 3 & 1 & 2 & 1 & 3 & 1 & 2 & 1 & 3 & 1 & 2 & 1 & 3\end{array}$

Figure 8: A packing 15-coloring of $P_{13} \square C_{16}$

If the conjecture holds, the vertices of the class $X_{1}$ can be fixed and therefore the backtracking is capable to provide results for much larger graph. In order to provide lower bounds for graphs of moderate size we therefore applied backtracking with no additional constraints, while for larger graphs the vertices of the class $X_{1}$ were fixed. The results of these computations are summarized in Table 1. The results in the table are of two types: exact values and upper bounds. Some of the upper bounds are exact values of $\chi_{\rho}$ if the Conjecture 3.2 holds. If a value $k$ in the table is exact, that means that a packing $k$-coloring for the graph of interest is found and that the backtracking procedure confirmed that a packing $(k-1)$-coloring does not exist. An upper bound $k$ means that a packing $k$-coloring for the graph of interest exists, but we could not prove that a packing $(k-1)$-coloring does not exist. On the other hand, if an upper bound $k$ is marked with asterisk, the backtracking proved that a packing $(k-1)$-coloring with the vertices of the class $X_{1}$ fixed as stated in the conjecture does not exist. 


\begin{tabular}{|c||c|c|c|c|c|c|c|c|c|c|c|c|c|}
\hline$m \backslash n$ & 6 & 7 & 8 & 9 & 10 & 11 & 12 & 13 & $14-15$ & $16-24$ & $25-27$ & $28-41$ & $>41$ \\
\hline 6 & 8 & 9 & 9 & 9 & 9 & 9 & 10 & 10 & 10 & 10 & 10 & 10 & 10 \\
\hline 7 & & 9 & 9 & 9 & 10 & 10 & 10 & 10 & 10 & $\leq 11^{*}$ & $\leq 11^{*}$ & $\leq 11^{*}$ & $\leq 11^{*}$ \\
\hline 8 & & & 9 & 10 & 10 & 10 & $\leq 11^{*}$ & $\leq 11^{*}$ & $\leq 11^{*}$ & $\leq 11^{*}$ & $\leq 11^{*}$ & $\leq 12$ & $\leq 12$ \\
\hline 9 & & & & 10 & $\leq 11^{*}$ & $\leq 11^{*}$ & $\leq 11^{*}$ & $\leq 11^{*}$ & $\leq 11^{*}$ & $\leq 12^{*}$ & $\leq 12^{*}$ & $\leq 12^{*}$ & $\leq 13$ \\
\hline 10 & & & & & $\leq 11^{*}$ & $\leq 11^{*}$ & $\leq 11^{*}$ & $\leq 12^{*}$ & $\leq 12^{*}$ & $\leq 12^{*}$ & $\leq 14$ & $\leq 14$ & $\leq 14$ \\
\hline 11 & & & & & & $\leq 11^{*}$ & $\leq 12^{*}$ & $\leq 12^{*}$ & $\leq 14$ & $\leq 14$ & $\leq 14$ & $\leq 14$ & $\leq 14$ \\
\hline 12 & & & & & & & $\leq 12^{*}$ & $\leq 12^{*}$ & $\leq 15$ & $\leq 15$ & $\leq 15$ & $\leq 15$ & $\leq 15$ \\
\hline 13 & & & & & & & & $\leq 13$ & $\leq 15$ & $\leq 15$ & $\leq 15$ & $\leq 15$ & $\leq 15$ \\
\hline
\end{tabular}

Table 1: Packing chromatic numbers and bounds for $P_{m} \square P_{n}$.

\section{Subgraphs of $\mathbb{Z} \square \mathbb{Z} \square \boldsymbol{P}_{2}$}

It is known that $\chi_{\rho}\left(\mathbb{Z}^{3}\right)=\infty$ [4]. Moreover even the packing chromatic number of $\mathbb{Z} \square \mathbb{Z} \square P_{2}$ is unbounded [3]. On the other hand, it was proved that $\chi_{\rho}(G \square \mathbb{Z})<\infty$ for any finite graph $G[3]$.

Hence, it is worthy to study the packing chromatic number of some infinite subgraphs of $\mathbb{Z} \square \mathbb{Z} \square P_{2}$. In particular we considered $C_{4} \square \mathbb{Z}, C_{6} \square \mathbb{Z}, C_{8} \square \mathbb{Z}, C_{10} \square \mathbb{Z}, C_{12} \square \mathbb{Z}$, and $P_{2} \square P_{3} \square \mathbb{Z}$. We were able to obtain exact results for the packing chromatic number of $C_{4} \square \mathbb{Z}$, while for the other families some partial results and bounds were found.

\section{Proposition 4.1.}

(i) $\chi_{\rho}\left(C_{4} \square \mathbb{Z}\right)=9$,

(ii) $\chi_{\rho}\left(C_{6} \square \mathbb{Z}\right) \leq 13$,

(iii) $\chi_{\rho}\left(C_{8} \square \mathbb{Z}\right) \leq 15$,

(iv) $\chi_{\rho}\left(C_{10} \square \mathbb{Z}\right) \leq 22$,

(v) $\chi_{\rho}\left(C_{12} \square \mathbb{Z}\right) \leq 17$,

(vi) $\chi_{\rho}\left(P_{2} \square P_{3} \square \mathbb{Z}\right) \leq 18$.

Proof. The upper bounds follow from the packing 9-coloring of $C_{4} \square C_{16}$ and from the packing 15-coloring of $C_{8} \square C_{24}$ depicted in Fig 9 and Fig 10, respectively. The packing 13-coloring of $C_{6} \square C_{48}$, the packing 22-coloring of $C_{10} \square C_{48}$, the packing 17-coloring of $C_{12} \square C_{48}$ and the packing 18-coloring of $P_{2} \square P_{3} \square C_{48}$ can be obtained from the authors or from the web page presented in the concluding remark.

$\begin{array}{llllllllllllllll}1 & 4 & 1 & 6 & 1 & 5 & 1 & 8 & 1 & 4 & 1 & 6 & 1 & 5 & 1 & 9 \\ 2 & 1 & 3 & 1 & 2 & 1 & 3 & 1 & 2 & 1 & 3 & 1 & 2 & 1 & 3 & 1 \\ 1 & 5 & 1 & 7 & 1 & 4 & 1 & 9 & 1 & 5 & 1 & 7 & 1 & 4 & 1 & 8 \\ 3 & 1 & 2 & 1 & 3 & 1 & 2 & 1 & 3 & 1 & 2 & 1 & 3 & 1 & 2 & 1\end{array}$

Figure 9: A packing 9-coloring of $C_{4} \square C_{16}$

The lower bound for $C_{4} \square P_{10}$ is obtained by using the backtracking procedure which confirms that 8-coloring of $C_{4} \square P_{10}$ does not exist.

Note that a coloring of $G$ which provides the upper bound in Proposition 4.1 has the vertices of the class $X_{1}$ distributed such that the cardinality of $X_{1}$ equals the independence number of $G$. We therefore generalize Conjecture 3.2 as follows.

Let $X_{m}$ denote $P_{m}$ or $C_{m}$. 


$\begin{array}{rrrrrrrrrrrrrrrrrrrrrrrr}1 & 13 & 1 & 8 & 1 & 4 & 1 & 5 & 1 & 9 & 1 & 4 & 1 & 5 & 1 & 8 & 1 & 4 & 1 & 5 & 1 & 9 & 1 & 4 \\ 3 & 1 & 2 & 1 & 3 & 1 & 2 & 1 & 3 & 1 & 2 & 1 & 3 & 1 & 2 & 1 & 3 & 1 & 2 & 1 & 3 & 1 & 2 & 1 \\ 1 & 6 & 1 & 7 & 1 & 12 & 1 & 14 & 1 & 6 & 1 & 7 & 1 & 13 & 1 & 15 & 1 & 6 & 1 & 7 & 1 & 10 & 1 & 5 \\ 2 & 1 & 3 & 1 & 2 & 1 & 3 & 1 & 2 & 1 & 3 & 1 & 2 & 1 & 3 & 1 & 2 & 1 & 3 & 1 & 2 & 1 & 3 & 1 \\ 1 & 4 & 1 & 9 & 1 & 5 & 1 & 4 & 1 & 8 & 1 & 5 & 1 & 4 & 1 & 9 & 1 & 5 & 1 & 4 & 1 & 8 & 1 & 11 \\ 3 & 1 & 2 & 1 & 3 & 1 & 2 & 1 & 3 & 1 & 2 & 1 & 3 & 1 & 2 & 1 & 3 & 1 & 2 & 1 & 3 & 1 & 2 & 1 \\ 1 & 5 & 1 & 15 & 1 & 6 & 1 & 7 & 1 & 10 & 1 & 11 & 1 & 6 & 1 & 7 & 1 & 12 & 1 & 14 & 1 & 6 & 1 & 7 \\ 2 & 1 & 3 & 1 & 2 & 1 & 3 & 1 & 2 & 1 & 3 & 1 & 2 & 1 & 3 & 1 & 2 & 1 & 3 & 1 & 2 & 1 & 3 & 1\end{array}$

Figure 10: A packing 15-coloring of $C_{8} \square C_{24}$

Conjecture 4.2. Let $n \geq 4$ and let $\chi_{\rho}\left(X_{m} \square P_{\ell} \square P_{n}\right)=k$. Then there exists a packing $k$-coloring of $X_{m} \square P_{\ell} \square P_{n}$ such that $\left|X_{1}\right|=\alpha\left(X_{m} \square P_{\ell} \square P_{n}\right)$.

Analogous as in Section 3 we therefore applied backtracking with no additional constraints for graphs of moderate size, while for larger graphs the vertices of the class $X_{1}$ were fixed. The packing colorings of the graphs of interest can be obtained from the authors or from the web page presented in the concluding remark. The results of these computations are summarized in Table 2, where an upper bound $k$ marked with asterisk means that the backtracking proved that a packing $(k-1)$-coloring with the vertices of the class $X_{1}$ fixed as stated in the conjecture does not exist.

\begin{tabular}{|c||c|c|c|c|c|c|c|c|c|c|c|c|c|c|}
\hline$m \backslash n$ & 2 & 3 & 4 & 5 & 6 & 7 & 8 & 9 & 10 & 11 & $12-15$ & $16-18$ & $19-34$ & $>34$ \\
\hline 4 & 5 & 5 & 7 & 7 & 7 & 7 & 8 & 8 & 9 & 9 & 9 & 9 & 9 & 9 \\
\hline 6 & 5 & 8 & 8 & 8 & 10 & 10 & 11 & 11 & 11 & 12 & $\leq 12^{*}$ & $\leq 12^{*}$ & $\leq 13$ & $\leq 13$ \\
\hline 8 & 7 & 7 & 9 & 9 & 10 & 10 & 11 & $\leq 12^{*}$ & $\leq 12^{*}$ & $\leq 13^{*}$ & $\leq 13^{*}$ & $\leq 14$ & $\leq 14$ & $\leq 15$ \\
\hline \hline$P_{2} \square P_{3}$ & 5 & 8 & 8 & 10 & 10 & 11 & $\leq 12^{*}$ & $\leq 12^{*}$ & $\leq 14$ & $\leq 15$ & $\leq 18$ & $\leq 18$ & $\leq 18$ & $\leq 18$ \\
\hline
\end{tabular}

Table 2: Packing chromatic numbers for $C_{m} \square P_{n}$ and $P_{2} \square P_{3} \square P_{n}$ (below).

\section{Concluding remark}

All obtained packing colorings as well as the graph $D_{6}$ can be obtained from the authors or directly from the web page http://matematika-racunalnistvo. fnm. uni-mb.si/personal/vesel/constructions.aspx.

\section{Acknowledgments}

We would like to thank the referees for their careful reading and helpful suggestions.

\section{References}

[1] B. Brešar, S. Klavžar and D. F. Rall, On the packing chromatic number of Cartesian products, hexagonal lattice, and trees, Discrete Appl. Math. 155 (2007), 2303-2311.

[2] J. Ekstein, J. Fiala, P. Holub and B. Lidický, The packing chromatic number of the square lattice is at least 12 , manuscript. 
[3] J. Fiala, S. Klavžar and B. Lidický, The packing chromatic number of infinite product graphs, European J. of Combin. 30 (2009), 1101-1113.

[4] A. S. Finbow and D. F. Rall On the packing chromatic number of some lattices, Discrete Appl. Math. 158 (2010), 1224-1228.

[5] A. Gamst, Some lower bounds for a class of frequency assignment problems, IEEE Trans. Veh. Technol. 35 (1986), 8-14.

[6] W. Goddard, S. M. Hedetniemi, S. T. Hedetniemi, J. M. Harris and D. F. Rall, Broadcast chromatic numbers of graphs, Ars Combin. 86 (2008), 33-49.

[7] P. Holub and R. Soukal, A note on packing chromatic number of the square lattice, Electron. J. Combin. 17 (2010), R\#17.

[8] W. Imrich and S. Klavžar, Product Graphs: Structure and Recognition, Wiley-Interscience, New York, 2000.

[9] S. Klavžar and A. Vesel, Computing graph invariants on rotagraphs using dynamic algorithm approach: the case of $(2,1)$-colorings and independence numbers, Discrete Appl. Math. 129 (2003), 449-460.

[10] A. Nijenhuis and H. S. Wilf, Combinatorial Algorithms, Academic Press, 1978. 